\title{
Age Determination of Blood-Stained Fingerprints Using Visible Wavelength Reflectance Hyperspectral Imaging
}

\author{
Samuel Cadd, Bo Li@, Peter Beveridge, William T. O'Hare and Meez Islam * \\ School of Science Engineering and Design, Teesside University, Borough Road, Middlesbrough TS1 3BA, UK; \\ cadd.sam@gmail.com (S.C.); bo.li@emr.ac.uk (B.L.); p.beveridge@tees.ac.uk (P.B.); \\ w.t.o.hare@gmail.com (W.T.O.) \\ * Correspondence: m.islam@tees.ac.uk; Tel.: +44-16-4234-2410
}

Received: 21 October 2018; Accepted: 27 November 2018; Published: 29 November 2018

\begin{abstract}
The ability to establish the exact time a crime was committed is one of the fundamental aims of forensic science. The analysis of recovered evidence can provide information to assist in age determination, such as blood, which is one of the most commonly encountered types of biological evidence and the most common fingerprint contaminant. There are currently no accepted methods to establish the age of a blood-stained fingerprint, so progress in this area would be of considerable benefit for forensic investigations. A novel application of visible wavelength reflectance, hyperspectral imaging (HSI), is used for the detection and age determination of blood-stained fingerprints on white ceramic tiles. Both identification and age determination are based on the unique visible absorption spectrum of haemoglobin between 400 and $680 \mathrm{~nm}$ and the presence of the Soret peak at $415 \mathrm{~nm}$. In this study, blood-stained fingerprints were aged over 30 days and analysed using HSI. False colour aging scales were produced from a 30-day scale and a $24 \mathrm{~h}$ scale, allowing for a clear visual method for age estimations for deposited blood-stained fingerprints. Nine blood-stained fingerprints of varying ages deposited on one white ceramic tile were easily distinguishable using the 30-day false colour scale.
\end{abstract}

Keywords: fingerprints; blood detection; age determination; hyperspectral imaging

\section{Introduction}

The reliable and accurate determination of when a crime was committed is one of the fundamental aims of forensic research. The analysis of recovered evidence from a crime scene can provide information to assist in this determination. At violent crime scenes, blood is one of the most commonly encountered types of biological evidence [1] and is the most common fingerprint contaminant [2]. The initial objective when dealing with suspected blood evidence is to conclusively establish that the substance is actually blood [3]. Dark substrates can pose considerable problems, due to the low contrast between the substrate and the fingerprint, due to the high amount of incident light absorbed by the surface [3]. Other colours or patterns that are particularly similar to the stain can also cause issues for identification through visual examination alone. Presumptive tests are therefore used as part of the current forensic workflow to indicate the presence of blood [2]. Despite a high sensitivity to blood, these wet chemical tests are not specific to blood and can generate false positives [2]. Wet chemical testing can also contaminate the stain, potentially having a detrimental effect on subsequent DNA analysis [4]. Previous and current research has therefore focused on the development of alternate methods for the non-destructive identification of blood [1,5-13].

The ability to establish the age of a fingerprint is a highly relevant factor in criminal investigations [14]. Convictions can largely depend on the ability to show whether a fingerprint 
was deposited at the time a crime was committed or from a previous legitimate visit, as is often claimed by the defence team $[15,16]$. There are currently no accepted analytical methods for reliably establishing a time frame when a fingerprint was deposited, and speculation around age is subject to considerable error [17]. This is primarily due to the unreliability of previously proposed methods [14]. Successful identification of blood and estimation of the age of a blood-stained fingerprint could provide the first indication to investigators as to when a crime was committed [18]. This could be especially beneficial if a blood-stained fingerprint is the only evidence available.

Early research made small steps forward in scientific knowledge, including exploring the solubility of blood in water over time, as the solubility rapidly drops over time before decreasing more slowly [19]. Other techniques have also furthered the understanding of the effect of time on blood stains, including the use of oxygen electrodes to establish the changes that occur to the oxyhaemoglobin-haemoglobin ratio in blood stains [20]. Other methods have also been explored, including the analysis of RNA degradation to establish the age of blood stains [21-23]. Spectroscopic methods were explored over the past decade, including atomic force microscopy, which was used to explore the elasticity of blood stains on glass slides through coagulation over time [11]. A clear increase in the stiffness of blood samples over time was identified, although several limitations to the method were identified. Research exploring electron spin resonance spectroscopy established a relationship between the electron paramagnetic resonance (EPR) of ferric non-heme species and the number of days from bleeding [24]. An error range within $25 \%$ of the actual number of days was obtained under controlled conditions, but environmental factors, such as light exposure and temperature, had effects on the analysis of EPR-active compounds.

It has been established that the colour of a blood stain changes from red to brown over time [6]. This indicates that optical methods could be used to quantify the colour of blood stains. This was first explored using the reflectance spectra of blood stains, whereby the effects of environmental variables on the colour of the blood stain were recognised [25]. Further research quantified absorption bands independent of the amount of blood present as a possible approach for age determination [26] or for the use of a small spectral window [27].

Previous research has clearly established changes to the physical and chemical properties of blood over time [6]. The optimum technique will require a high selectivity to blood; a high level of sensitivity, even with diluted blood; and a high level of precision to determine the age of a blood stain or blood-stained fingerprint in practice. One method is the use of visible wavelength hyperspectral imaging. This was first reported for the detection and age determination of horse blood stains between 442 and $585 \mathrm{~nm}$ as proof of concept research [28]. The determination of age was obtained through linear discriminant analysis from data based on the progressive changes in the absorption spectra over time as the composition of the blood stain altered. This approach used training and test datasets from the same blood stain in order to determine the age with a high level of accuracy. With different blood stains, the accuracy was considerably lower, although this research demonstrated the potential of the method to establish an age estimation non-destructively. A similar method by a different research group also successfully demonstrated the identification of blood stains $[29,30]$. The proposed method allowed for rapid, non-destructive presumptive blood stain detection. Other research has explored forensic traces across a range of substrates [31]. Most recently, a new blood stain identification and age determination approach was proposed based on the Soret $\gamma$ band absorption in haemoglobin $[3,32]$ and indicated a higher sensitivity and specificity for the detection and identification of blood stains over previously proposed methods [3].

Previous research has identified the need for a non-contact and non-destructive method for the determination of the age of a blood-stained fingerprint. An ideal method should function across a practical range of ages of blood, have a high specificity to blood so as to prevent false positives, and have a clear and accurate method for determining the age of a blood-stained fingerprint, so as to allow for reliable age estimations.

The visible wavelength hyperspectral imaging method proposed in this paper meets all of these requirements. In this study, we present a novel application of visible wavelength hyperspectral imaging 
(HSI) based on the absorption spectrum of haemoglobin between 400 and $680 \mathrm{~nm}$ for the non-contact, non-destructive detection, identification and age determination of blood-stained fingerprints on white tiles. False colour scales are presented based on the ratio of the $525 \mathrm{~nm}$ peak to the trough at $550 \mathrm{~nm}$ in the absorption spectrum. The 30-day colour scale is demonstrated with nine blood-stained fingerprints deposited on a single white tile to demonstrate the effectiveness of such a method for age determination.

This work follows on from $[33,34]$ where hyperspectral imaging was used for the first time to detect, identify, and visualise ridge detail in blood-stained fingerprints across a wide range of substrates. The research presented in this paper again demonstrates the potential of HSI, through successful non-destructive detection, identification, and age determination of blood-stained fingerprints.

\section{Materials and Methods}

\subsection{Production of Blood-Stained Fingerprints}

Horse blood was used to produce blood-stained fingerprints in this study and was deposited into a Petri dish containing a small sponge. The right middle finger was pressed against the sponge to evenly coat the digit, and the blood-stained fingerprint was then deposited onto the white tile. The fingerprints were left to age under controlled conditions in an environmental chamber at $23-24{ }^{\circ} \mathrm{C}$ (Qualicool LR202, LTE Scientific, Oldham, UK) between analyses.

\subsection{HSI System}

The HSI system used in this study had the same setup as that detailed in $[3,33,34]$, consisting of a liquid crystal tuneable filter (LCTF) coupled to a 2.3 megapixel Point Grey camera and a light source for scene illumination. The light source was comprised of two $40 \mathrm{~W}$ LEDs-one violet giving an output at $410 \mathrm{~nm}$ and one white giving an output between 450 and $700 \mathrm{~nm}$. Control of the LCTF and image capture was performed using custom developed software written in C++ (Microsoft, Redmond, WA, USA). Images were captured between 400 and $680 \mathrm{~nm}$ with spectral sub-sampling at $5 \mathrm{~nm}$ intervals, resulting in an image cube at 56 wavelengths for each scan. Spectra from the image cube were subsequently analysed using custom routines developed in Visual Studio (Microsoft, USA) and Spyder (Python, Wilmington, DE, USA). The time required to acquire and process each image was approximately $30 \mathrm{~s}$.

\subsection{Hyperspectral Reflectance Image Acquisition and Pre-Processing}

The hyperspectral reflectance measurements were made following the method detailed in [3] and $[33,34]$. A reference image $\left(\mathrm{R}_{0}\right)$ was obtained using a blank ceramic tile. This image was recorded in a $5 \mathrm{~nm}$ series of 56 discrete wavelengths between 400 and $680 \mathrm{~nm}$. The sample image $\left(R_{s}\right)$ was recorded at the same wavelengths under the same illumination conditions and integration time settings on the camera. The hyperspectral reflectance image $(\mathrm{R})$ consisted of a data cube of $1280 \times 1024$ pixel values at 56 discrete wavelengths.

\subsection{Criteria for the Identification of Blood Stains}

The blood reflectance spectrum in the visible region is dominated by the presence of haemoglobin [28,32]. The spectrum contains a strong narrow absorption at $415 \mathrm{~nm}$ called the Soret or $\gamma$ band with two weaker and broader absorptions between 500 and $600 \mathrm{~nm}$ known as the $\beta$ and $\alpha$ bands [3]. The Soret band results in the distinctive red colour of blood, due to the absorption in the blue part of the visible spectrum. Other red substances also absorb between 400 and $680 \mathrm{~nm}$ in the blue region, although these absorption features tend to not be centred at $415 \mathrm{~nm}$ and are much broader. This is the basis of the methodology used to identify and discriminate blood stains from other similarly coloured substances. Further information is detailed in [3]. From the reflectance images obtained, the pixels which satisfied the criteria were marked in black, whilst all other pixels were marked in white. 
This allowed regions of the image where the blood-stained fingerprint was present to be identified, as well as clear distinction of the ridge detail.

\subsection{Age Determination Methodology for Blood-Stained Fingerprints}

The age of the blood-stained fingerprints was explored through the effect of time on the composition of the fingerprint. This has already been established as the only potentially viable method for age determination, due to the numerous variables that affect fingerprint composition over time [14]. This research explored the compositional changes that occur within a blood-stained fingerprint using hyperspectral imaging. After deposition of a blood-stained fingerprint, specific chemical changes occur which result in a colour change from bright red to dark brown. This is attributed to the complete oxidation of haemoglobin $(\mathrm{Hb})$ to oxy-haemoglobin $\left(\mathrm{HbO}_{2}\right)$, which then auto-oxidises to met-haemoglobin (met- $\mathrm{Hb})$ and denatures to hemichrome $(\mathrm{HC})[6,35]$, as shown in Figure 1. As this process occurs, the concentration of haemoglobin decreases, which can be observed in the visible spectrum through the decrease of the Soret band at $415 \mathrm{~nm}$, as shown in Figure 2.

After the HSI analysis, the absorption spectra of the blood-stained fingerprints were analysed, and the ratio of the peak at $525 \mathrm{~nm}$ to the trough at $550 \mathrm{~nm}$ was determined. This ratio was used to produce false colour Red-Green-Blue aging scales, as shown with ten false coloured fingerprints in Figure 3, with the values determining the values assigned to red, green, and blue in the image.

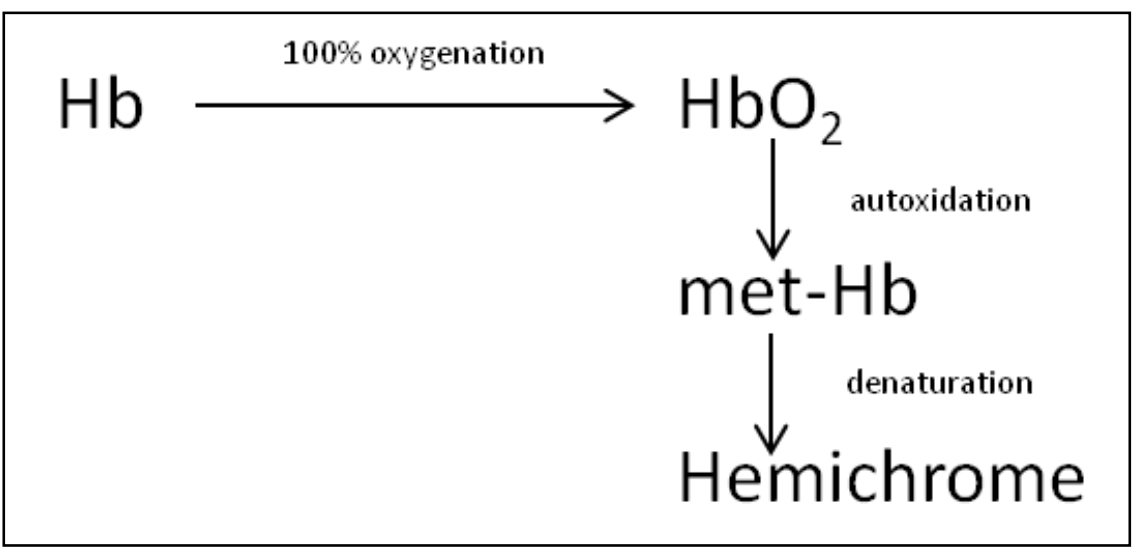

Figure 1. Reaction of haemoglobin in blood stains.

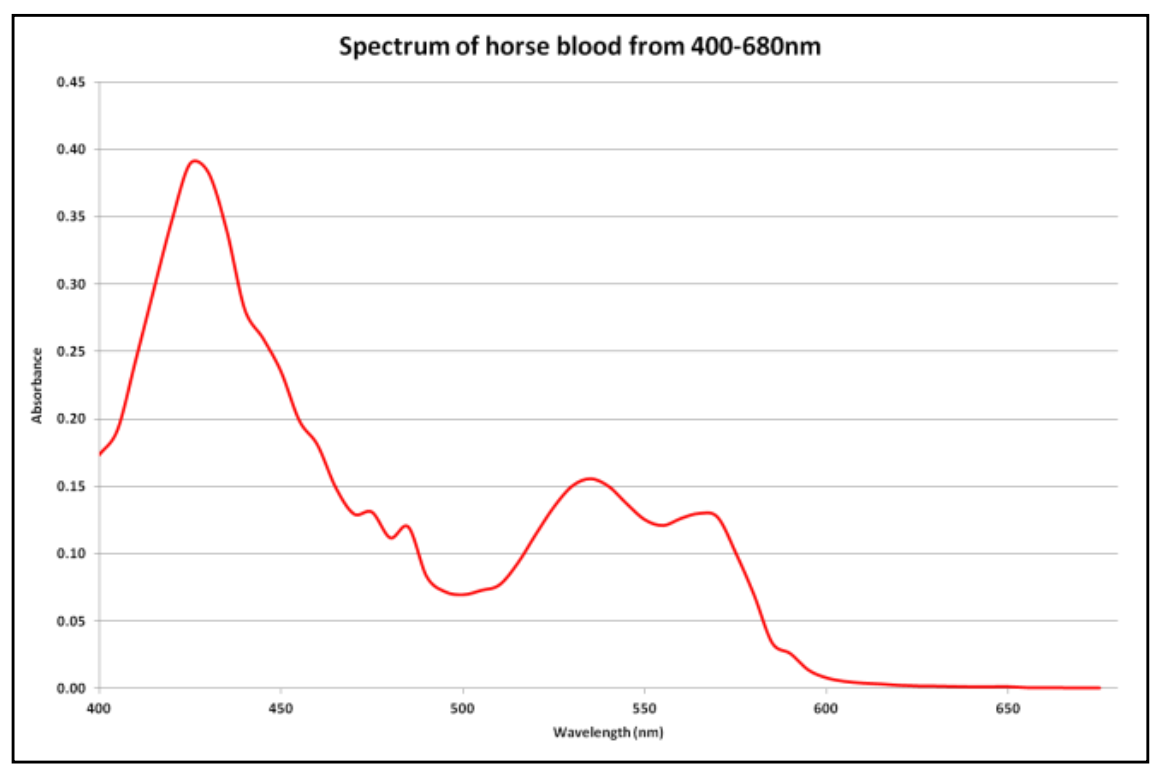

Figure 2. Spectrum of blood from $400-680 \mathrm{~nm}$. 


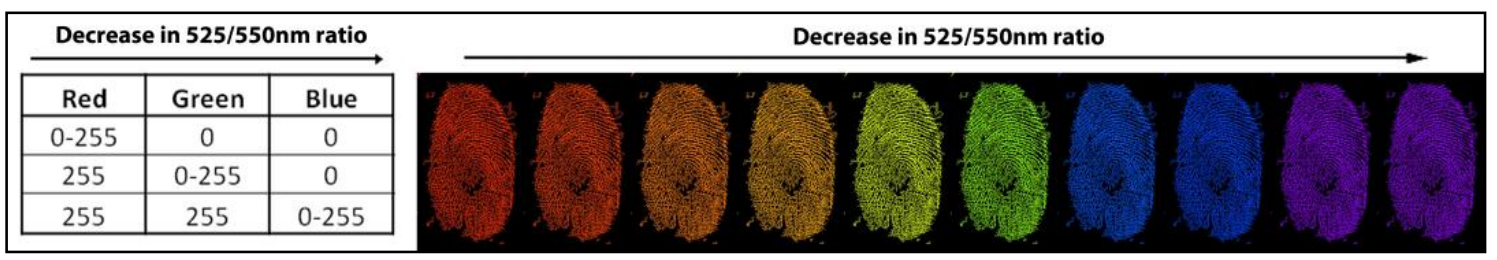

Figure 3. Use of Red-Green-Blue colours to produce false colour scales based on the 525/550 nm ratio.

\subsection{Digital Single Lens Reflex (DSLR) Setup}

The images used in this report were taken using a digital single lens reflex (DSLR) camera mounted on a Kaiser RS1 copy stand. The DSLR was a Canon EOS 700D which was fitted with a Canon Angle Finder C $90^{\circ}$ viewfinder with a 1.25-2.5 $\times$ optical magnification and a Canon TC-80N3 remote control external shutter release to avoid camera motion. Images were taken using two sizes of macro-lenses-a $50 \mathrm{~mm}$ lens for overview shots of the substrates and a $100 \mathrm{~mm}$ lens for high magnification macro-shots of individual fingerprints. The $50 \mathrm{~mm}$ lens was used as it is recognised as being generally equivalent to the view seen by the human eye [36]. The lenses used were a Canon EF $50 \mathrm{~mm}$ f2.5 macro lens and a Canon EF $100 \mathrm{~mm}$ f2.8L macro IS USM lens. Substrates were lit using oblique lighting from two Daylight Twist Portable Lamps with a white light output of $6500 \mathrm{~K}$.

\subsection{Age Estimation Intervals}

\subsubsection{Aging of Blood-Stained Fingerprints over 30 Days}

Deposited blood fingerprints were aged under controlled conditions in an environmental chamber at $23-24{ }^{\circ} \mathrm{C}$, as detailed in Section 2.1. Analyses were carried out daily for thirty days and false colour aging scales were produced after $7,16,24$, and 30 days.

\subsubsection{Aging of Blood-Stained Fingerprints over $24 \mathrm{~h}$}

Deposited blood fingerprints were also analysed over a $24 \mathrm{~h}$ period. Blood-stained fingerprints were deposited and analysed every hour for $12 \mathrm{~h}$. A second set of fingerprints was then deposited and left overnight for $12 \mathrm{~h}$. This set was then analysed from $12 \mathrm{~h}$ to $24 \mathrm{~h}$. The results from both sets were combined to produce the $24 \mathrm{~h}$ aging scale.

\subsubsection{Age Estimations of Nine Blood-Stained Fingerprints}

Nine blood-stained fingerprints were deposited between 0 to 28 days onto one white tile, as shown in Figure 4. These were deposited in a random arrangement on the white tile. The fingerprints were aged under controlled conditions in an environmental chamber at $23-24{ }^{\circ} \mathrm{C}$, as detailed in Section 2.1. Analyses were carried out using his, and the results were manually coloured using the 30-day false colour scale for a visual representation of the ages.

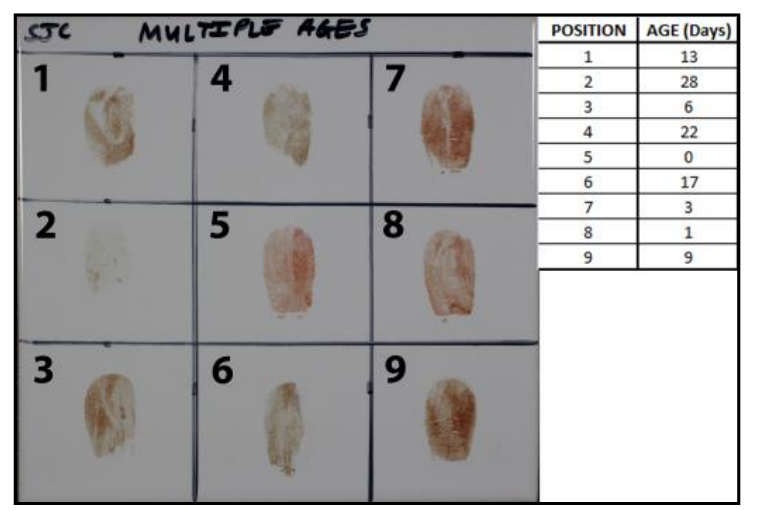

Figure 4. Nine blood-stained fingerprints from 0 to 28 days old. 


\section{Results}

\subsection{Aging of Blood-Stained Fingerprints over 30 Days}

Blood-stained fingerprints were successfully detected and identified using hyperspectral imaging for the full 30 days explored. Clear ridge detail was identified for all scans, a selection of which are shown in seven day increments from 0 to 28 days in Figure 5. The level of clear ridge detail observable even after 30 days demonstrates the advantage of HSI over existing chemical methods, as not only can blood be conclusively identified, as opposed to an indication as occurs with presumptive tests, but ridge detail is preserved and photographed for potential comparison in one step.

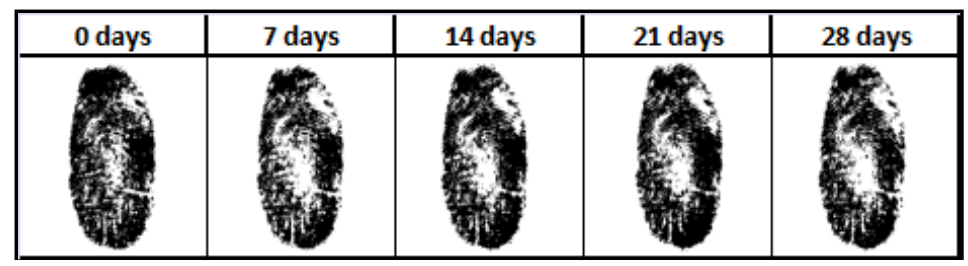

Figure 5. Visible ridge detail for blood-stained fingerprints with hyperspectral imaging (HSI) on day 0, $7,14,21$, and 28 .

The analysis of the absorption spectrum between 400 and $680 \mathrm{~nm}$ showed a clear decrease in the Soret band and the $\beta$ and $\alpha$ bands between 500 and $600 \mathrm{~nm}$ over the aging period. This change forms the basis for the age estimation methodology. False colour scales were produced to represent the changes in the 525/550 nm ratio over 30 days, as shown in Figure 6.

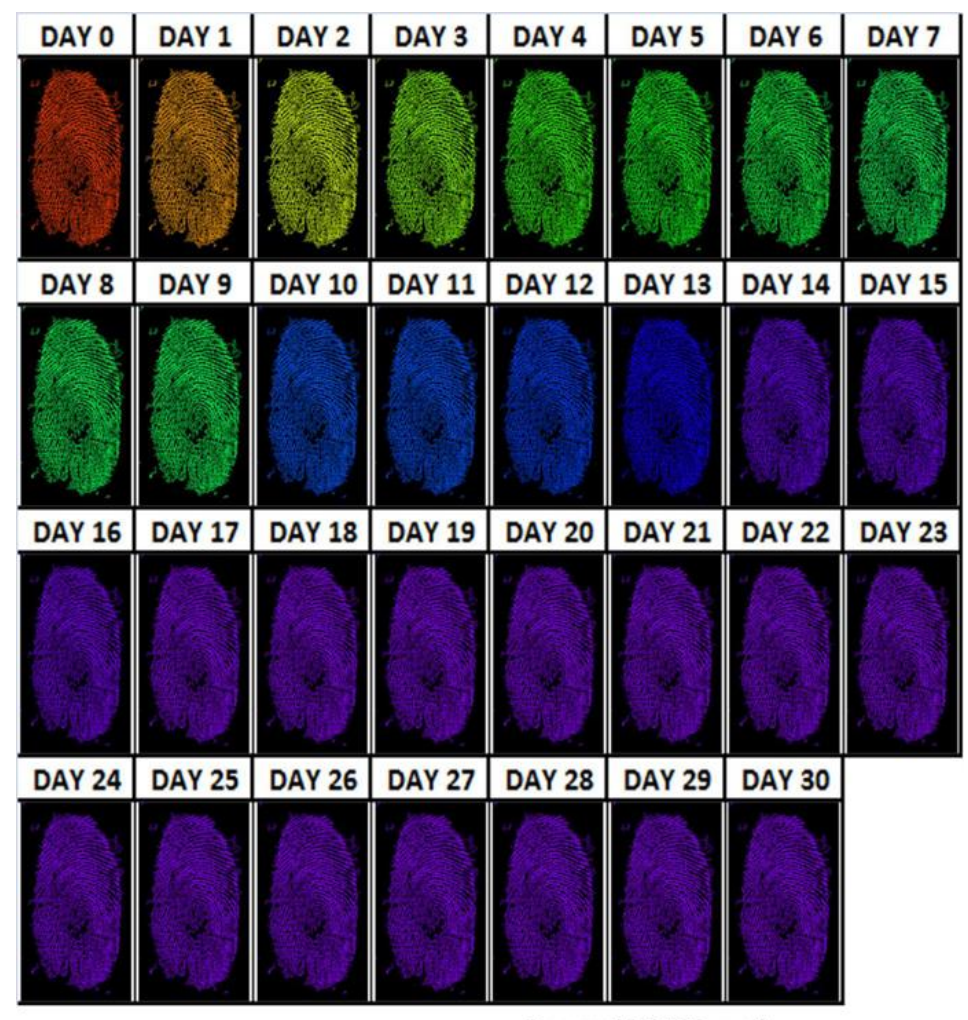

Decrease in $550 / 525 \mathrm{~nm}$ ratio

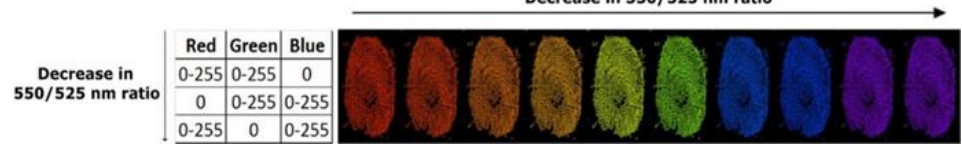

Figure 6. 30-day false colour aging scale. 


\subsection{Aging of Blood-Stained Fingerprints over $24 \mathrm{~h}$}

Blood-stained fingerprints were successfully detected using hyperspectral imaging for the full $24 \mathrm{~h}$ explored. Clear ridge detail was identified for all scans and the absorption spectrum was analysed to produce a false colour scale, as shown in Figure 7. This scale represents the changes that occurred in the absorption spectrum over the $24 \mathrm{~h}$ aging period, as shown in Figure 8. The logarithmic conversion also shown demonstrates the clear relationship between the 525/550 nm ratio and time.

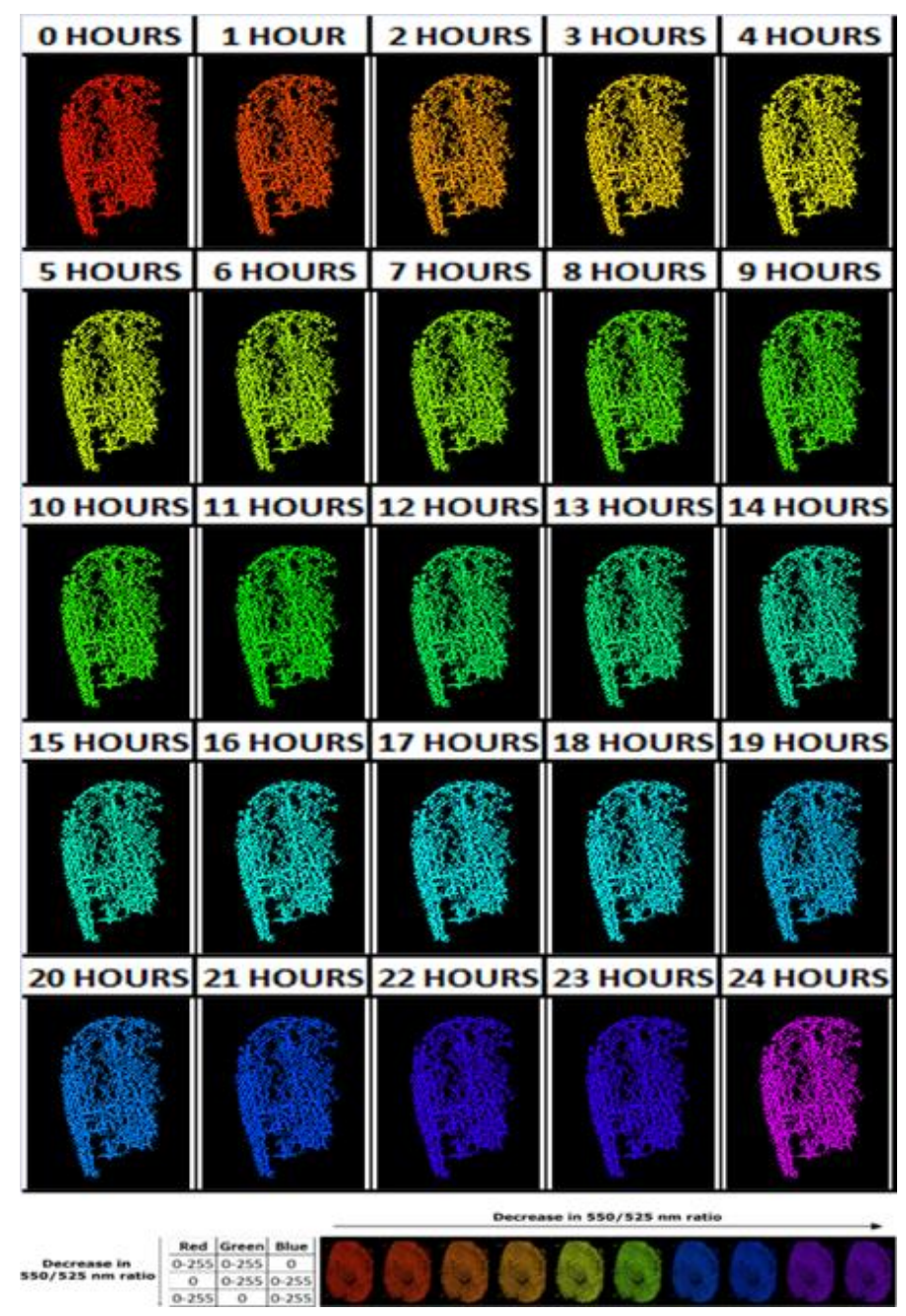

Figure 7. $24 \mathrm{~h}$ false colour aging scale.

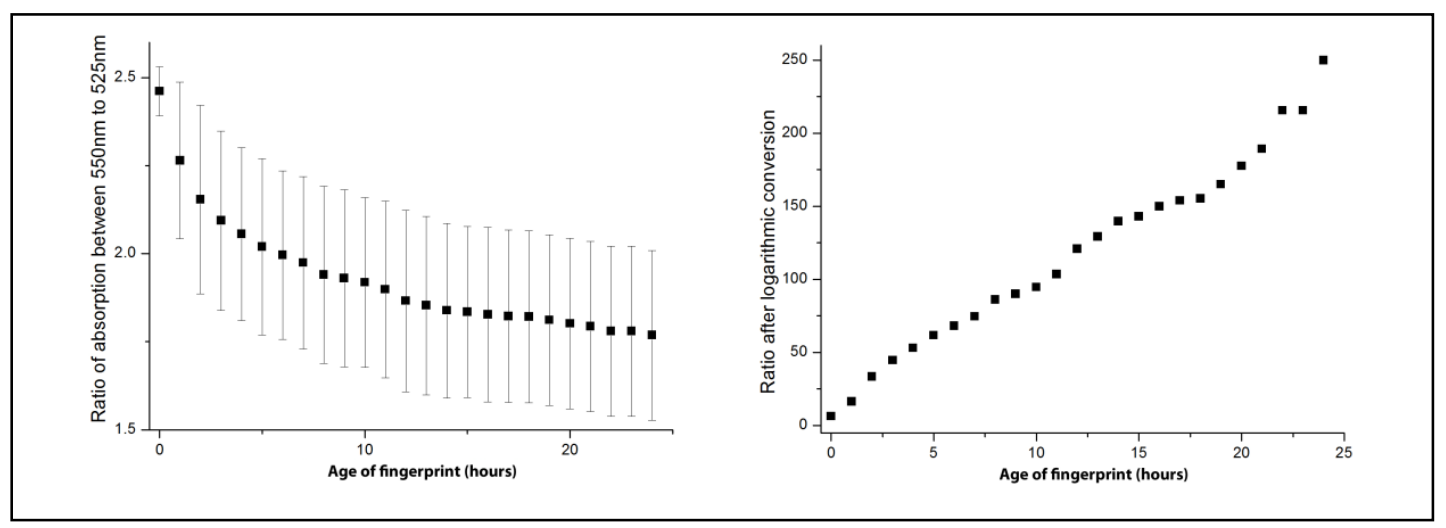

Figure 8. Effect of time on the absorption ratio of 550 and $525 \mathrm{~nm}$ (left) and the logarithmic conversion (right) over $24 \mathrm{~h}$. 


\subsection{Age Estimations of Nine Blood-Stained Fingerprints}

From a visual examination of the nine blood stains alone, it was very difficult to determine any significant differences that may allow for age estimations. The analysis carried out using HSI successfully detected and conclusively identified the blood ridge detail. Using the data obtained from the HSI analysis and the false colour scales produced, a clear visual representation of the different ages of the blood-stained fingerprints was produced, as shown in Figure 9. The DSLR images show minimal variation between the fingerprints. Using the false colour scales, all recently deposited fingerprints could be easily distinguished due to the significant differences in colour, such as fingerprints $5,8,7$, and 3 , which correspond to $0,1,3$, and 6 days respectively. After 14 days, the variation in the composition of the blood-stained fingerprint was less, so the difference between the colours was smaller and harder to distinguish by eye. This was apparent for fingerprints 2,4 , and 6 , which were all shades of purple, despite varying by eleven days. The use of this false colour method for age estimations is therefore most effective for blood-stained fingerprints deposited within 14 days, as the increased variation over the first seven days results in significant differences in the false colour images produced.

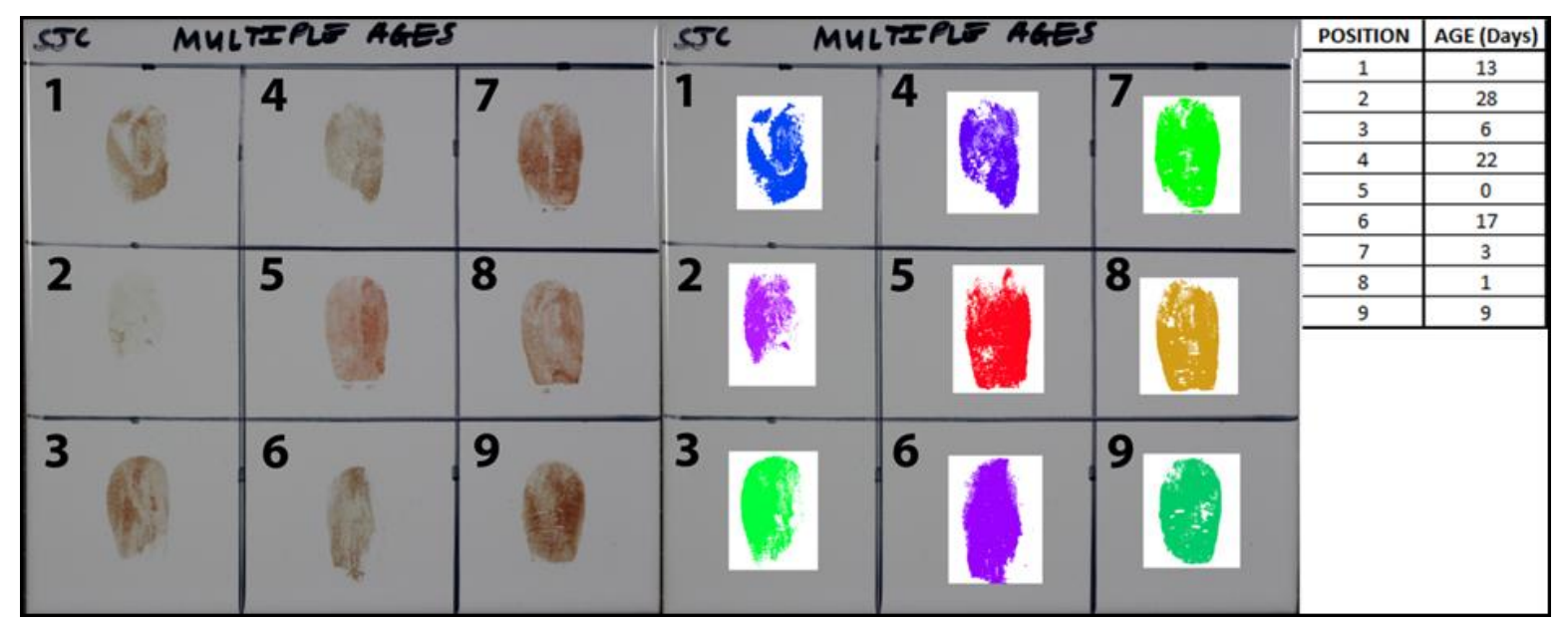

Figure 9. Digital single lens reflex (DSLR) (left) and false colour images manually coloured from HSI analysis (right) of blood-stained fingerprints based on a 30-day false colour scale.

\section{Discussion}

Results have been presented on the application of a visible wavelength reflectance hyperspectral imaging system for the detection of blood-stained fingerprints and the determination of the fingerprint age on white tiles. The method used for the detection and identification of blood as well as the age determination was based on use of the main narrow absorption peak in blood at approximately $415 \mathrm{~nm}$ (Soret band), and the ratio of the peak at $525 \mathrm{~nm}$ to the trough at $550 \mathrm{~nm}$ was also determined. This was used to produce a false colour aging scale over 30 days. A second false colour scale was also produced over $24 \mathrm{~h}$, allowing for highly accurate estimations of the age of freshly deposited blood-stained fingerprints.

Nine blood stains of varying ages deposited on one tile were able to be distinguished using the 30-day false colour scale. With visual examination alone, there were minimal differences between deposited fingerprints in the DSLR image, making it very difficult to establish any significant differences for age estimations. The analysis carried out using HSI successfully detected and identified the ridge detail. The analysis using the 30-day false colour scale established a clear visual representation of the different ages of the blood-stained fingerprints. Recently deposited fingerprints were easier to distinguish due to the greater differences in colour. After 14 days, this variation reduced, due to the slower changes in the blood composition, and the colours were slightly more difficult to distinguish. Previous research has demonstrated the success of using hyperspectral imaging as a method for establishing the age of blood stains, although a clear visual approach using false colour scales has not 
been carried out. The approach presented in this study demonstrates the considerable potential of HSI to both conclusively identify a blood-stained fingerprint and to determine its age.

\section{Conclusions}

A novel application of visible wavelength reflectance hyperspectral imaging (HSI) was used for both the detection and age determination of blood-stained fingerprints on white ceramic tiles. Both the identification of blood and the age determination were based on the unique visible absorption spectrum of haemoglobin between 400 and $680 \mathrm{~nm}$ and the presence of the Soret peak at $415 \mathrm{~nm}$. In the processed hyperspectral images, pixels where blood was identified were coloured black, whilst all other pixels were coloured white, thus enhancing the location of ridge detail in blood fingerprints.

Blood-stained fingerprints were aged over 30 days and analysed using HSI. From these results, a 30-day scale and a $24 \mathrm{~h}$ scale were produced, allowing for a clear visual method for age estimations of deposited blood-stained fingerprints. This was demonstrated with nine blood-stained fingerprints deposited on one white tile. From a visual examination using DSLR, no significant differences between the deposited fingerprints could be identified to allow for age estimations. The application of HSI demonstrated several advantages, as the analysis successfully detected and identified the blood ridge detail. Additionally, the application of the 30-day false colour scale allowed for the deposited blood-stained fingerprints to be coloured corresponding to their age. This identified clear distinctions between the nine blood-stained fingerprints in the false colour image produced, with the greatest differences in colour occurring among the most recently deposited fingerprints as the greatest differences in composition occur over the first $24 \mathrm{~h}$ after deposition [37,38].

Overall, HSI has significant benefits for both the detection and age determination of blood-stained fingerprints and blood stains. Preliminary work exploring both human and horse blood demonstrated minimal differences, indicating that the findings of this research are applicable to crime scenes involving human blood. Further work is required, however, to confirm that the age determination methodology demonstrated here is effective across both blood stains and blood-stained fingerprints with both human and horse blood. Large scale blind tests are also required to establish the effectiveness and reliability of the age determination method on white tiles, as well as other substrates, including other colours and porosities, and as a function of environmental variables, such as temperature and humidity. Previous work with this setup has already demonstrated the successful detection and identification of blood-stained fingerprints on a range of substrates [33,34]. A full comparison of the technique against existing chemical enhancement methods would be beneficial to allow a comparison of the sensitivity of the setups. Development of a more rugged instrument could allow for the production of a robust portable device for use at crime scenes, which would be particularly beneficial for criminal investigations. HSI could then be used for the detection and identification of both blood stains and blood-stained fingerprints, as well as for the reliable establishment of the age of a stain or fingerprint.

Author Contributions: S.C. and B.L. conducted the research presented in this study. M.I. and S.C. wrote the paper. M.I., W.T.O., P.B. and A.C. contributed to the development of the overall research design, provided guidance along the way and aided in the writing of the paper.

Funding: This research was partly funded by the Home Office through a SBRI phase 1 grant. S.C. would also like to acknowledge the Forensic Science Society, who kindly provided financial support through a Research Scholarship.

Acknowledgments: We would to thanks Steve Bleay at the Home Office, Centre for Applied Science and Technology (CAST).

Conflicts of Interest: The authors declare no conflict of interest.

\section{References}

1. Finnis, J.; Lewis, J.; Davidson, A. Comparison of methods for visualizing blood on dark surfaces. Sci. Justice 2013, 53, 178-186. [CrossRef] [PubMed]

2. Home Office CAST. Fingerprint Sourcebook, Chapter 3, 3.1 Acid Dyes, 1st ed.; Home Office: London, UK, 2013. 
3. Li, B.; Beveridge, P.; O'Hare, W.T.; Islam, M. The application of visible wavelength reflectance hyperspectral imaging for the detection and identification of blood stains. Sci. Justice 2014, 54, 432-438. [CrossRef] [PubMed]

4. Passi, N.; Garg, R.K.; Yadav, M.; Singh, R.S.; Kharoshah, M.A. Effect of luminol and bleaching agent on the serological and DNA analysis from bloodstain. Egypt. J. Forensic Sci. 2012, 2, 54-61. [CrossRef]

5. Anderson, S.; Howard, B.; Hobbs, G.R.; Bishop, C.P. A method for determining the age of a bloodstain. Forensic Sci. Int. 2005, 148, 37-45. [CrossRef] [PubMed]

6. Bremmer, R.H.; Nadort, A.; van Leeuwen, T.G.; van Gemert, M.J.C.; Aalders, M.C.G. Age estimation of blood stains by hemoglobin derivative determination using reflectance spectroscopy. Forensic Sci. Int. 2011, 206, 166-171. [CrossRef] [PubMed]

7. de Wael, K.; Lepot, L.; Gason, F.; Gilbert, B. In search of blood-Detection of minute particles using spectroscopic methods. Forensic Sci. Int. 2008, 180, 37-42. [CrossRef] [PubMed]

8. Lin, A.C.; Hsieh, H.S.; Li, T.; Linacre, A.; Lee, J.C. Forensic Applications of Infrared Imaging for the Detection and Recording of Latent Evidence. J. Forensic Sci. 2007, 52, 1148-1150. [CrossRef] [PubMed]

9. McLaughlin, G.; Sikirzhytski, V.; Lednev, I.K. Circumventing substrate interference in the Raman spectroscopic identification of blood stains. Forensic Sci. Int. 2013, 231, 157-166. [CrossRef] [PubMed]

10. Stoilovic, M. Detection of semen and blood stains using polilight as a light source. Forensic Sci. Int. 1991, 51, 289-296. [CrossRef]

11. Strasser, S.; Zink, A.; Kada, G.; Hinterdorfer, P.; Peschel, O.; Heckl, W.M.; Nerlich, A.G.; Thalhammer, S. Age determination of blood spots in forensic medicine by force spectroscopy. Forensic Sci. Int. 2007, 170, 8-14. [CrossRef] [PubMed]

12. Turrina, S.; Filippini, G.; Atzei, R.; Zaglia, E.; de Leo, D. Validation studies of rapid stain identification-blood (RSID-blood) kit in forensic caseworks. Forensic Sci. Int. Genet. Suppl. Ser. 2008, 1, 74-75. [CrossRef]

13. Wawryk, J.; Odell, M. Fluorescent identification of biological and other stains on skin by the use of alternative light sources. J. Clin. Forensic Med. 2005, 12, 296-301. [CrossRef] [PubMed]

14. Cadd, S.; Islam, M.; Manson, P.; Bleay, S. Fingerprint composition and aging: A literature review. Sci. Justice 2015. [CrossRef] [PubMed]

15. Gardner, T.; Anderson, T. Criminal Evidence: Principles and Cases, 7th ed.; Cengage Learning: Belmont, CA, USA, 2009.

16. Adebsi, S. Fingerprint Studies-The Recent Challenges And Advancements: A Literary View. Internet J. Boil. Anthr. 2008, 2,1-9.

17. Midkiff, C. Lifetime of a Latent Print How Long? Can You Tell? J. Forensic Identif. 1993, 43, 386-396.

18. Bremmer, R.H.; de Bruin, K.G.; van Gemert, M.J.C.; van Leeuwen, T.G.; Aalders, M.C.G. Forensic quest for age determination of bloodstains. Forensic Sci. Int. 2012, 216, 1-11. [CrossRef] [PubMed]

19. Schwarzacher, D. Determination of the age of bloodstains. Am. J. Police Sci. 1930. [CrossRef]

20. Matsuoka, T.; Taguchi, T.; Okuda, J. Estimation of bloodstain age by rapid determinations of oxyhemoglobin by use of oxygen electrode and total hemoglobin. Boil. Pharm. Bull. 1995, 18, 1031-1035. [CrossRef]

21. Bauer, M.; Polzin, S.; Patzelt, D. Quantification of RNA degradation by semi-quantitative duplex and competitive RT-PCR: A possible indicator of the age of bloodstains? Forensic Sci. Int. 2003, 138, 94-103. [CrossRef] [PubMed]

22. Bauer, M. RNA in forensic science. Forensic Sci. Int. Genet. 2007, 1, 69-74. [CrossRef] [PubMed]

23. Virkler, K.; Lednev, I.K. Analysis of body fluids for forensic purposes: From laboratory testing to non-destructive rapid confirmatory identification at a crime scene. Forensic Sci. Int. 2009, 188, 1-17. [CrossRef] [PubMed]

24. Fujita, Y.; Tsuchiya, K.; Abe, S.; Takiguchi, Y.; Kubo, S.; Sakurai, H. Estimation of the age of human bloodstains by electron paramagnetic resonance spectroscopy: Long-term controlled experiment on the effects of environmental factors. Forensic Sci. Int. 2005, 152, 39-43. [CrossRef] [PubMed]

25. Patterson, D. Use of reflectance measurements in assessing the colour changes of ageing bloodstains. Nature 1960, 20, 688-689. [CrossRef]

26. Kind, S.S.; Patterson, D.; Owen, G.W. Estimation of the age of dried blood stains by a spectrophotometric method. Forensic Sci. 1972, 1, 27-54. [CrossRef]

27. Blazek, V.; Lins, G. Spectroscopic age determination of blood stains: New technical aspects. Acta Med. Leg. Et Soc. 1982, 32, 613-616. 
28. Li, B.; Beveridge, P.; O'Hare, W.T.; Islam, M. The estimation of the age of a blood stain using reflectance spectroscopy with a microspectrophotometer, spectral pre-processing and linear discriminant analysis. Forensic Sci. Int. 2011, 212, 198-204. [CrossRef] [PubMed]

29. Janchaysang, S.; Sumriddetchkajorn, S.; Buranasiri, P. Tunable filter-based multispectral imaging for detection of blood stains on construction material substrates Part 1: Developing blood stain discrimination criteria. Appl. Opt. 2012, 51, 6984-6996. [CrossRef] [PubMed]

30. Janchaysang, S.; Sumriddetchkajorn, S.; Buranasiri, P. Tunable filter-based multispectral imaging for detection of blood stains on construction material substrates Part 2: Realization of rapid blood stain detection. Appl. Opt. 2013, 52, 4898-4910. [CrossRef] [PubMed]

31. Edelman, G.J.; Gaston, E.; van Leeuwen, T.G.; Cullen, P.J.; Aalders, M.C.G. Hyperspectral imaging for non-contact analysis of forensic traces. Forensic Sci. Int. 2012, 223, 28-39. [CrossRef] [PubMed]

32. Li, B.; Beveridge, P.; O'Hare, W.T.; Islam, M. The age estimation of blood stains up to 30 days old using visible wavelength hyperspectral image analysis and linear discriminant analysis. Sci. Justice 2013, 53, $270-277$. [CrossRef] [PubMed]

33. Cadd, S.; Li, B.; Beveridge, P.; O’Hare, W.T.; Campbell, A.; Islam, M. Non-contact detection and identification of blood stained fingerprints using visible wavelength reflectance hyperspectral imaging: Part 1. Sci. Justice 2016, 56, 181-190. [CrossRef] [PubMed]

34. Cadd, S.; Li, B.; Beveridge, P.; O'Hare, W.T.; Campbell, A.; Islam, M. Non-contact detection and identification of blood stained fingerprints using visible wavelength reflectance hyperspectral imaging: Part II effectiveness on a range of substrates. Sci. Justice 2016, 56, 191-200. [CrossRef] [PubMed]

35. Bremmer, R.H.; de Bruin, D.M.; de Joode, M.; jan Buma, W.; van Leeuwen, T.G.; Aalders, M.C.G. Biphasic oxidation of oxy-hemoglobin in bloodstains. PLoS ONE 2011, 6, e21845. [CrossRef] [PubMed]

36. Langford, M.; Fox, A.; Smith, R.S. Langford's Basic Photography, 8th ed.; Focal Press: Oxford, UK, 2009.

37. Cadd, S.; Bleay, S.; Sears, V. Evaluation of the solvent black 3 fingermark enhancement reagent: Part 2-Investigation of the optimum formulation and application parameters. Sci. Justice 2013, 53, 131-143. [CrossRef] [PubMed]

38. Archer, N.; Charles, Y.; Elliott, J.; Jickells, S. Changes in the lipid composition of latent fingerprint residue with time after deposition on a surface. Forensic Sci. Int. 2005, 154, 224-239. [CrossRef] [PubMed] 